\title{
Challenging dicta in thoracic surgery
}

\author{
Varun Puri, MD, MSCI
}

From the Division of Cardiothoracic Surgery, Washington University School of Medicine, St. Louis, Mo. Disclosures: Author has nothing to disclose with regard to commercial support.

Received for publication Feb 3, 2017; accepted for publication Feb 6, 2017; available ahead of print March 5, 2017.

Address for reprints: Varun Puri, MD, MSCI, Division of Cardiothoracic Surgery, Washington University School of Medicine, Campus Box 8234, 660 S. Euclid Ave, St. Louis, MO 63110 (E-mail: puriv@ wudosis.wustl.edu). J Thorac Cardiovasc Surg 2017;153:e119-20 0022-5223/ $\$ 36.00$

Copyright (C) 2017 by The American Association for Thoracic Surgery http://dx.doi.org/10.1016/j.jtcvs.2017.02.006

Guo and colleagues ${ }^{1}$ present a patient with symptomatic bilateral apical bullous disease who underwent bilateral lung wedge resection via a uniportal subxiphoid approach. Building on previous experience, the authors highlight that it is safe to (1) perform bilateral thoracoscopic surgery, (2) avoid general anesthetic, (3) omit pleurodesis for bleb resection, and (4) circumvent chest drainage after thoracoscopic surgery. Doing so potentially simplifies perioperative care and streamlines postoperative recovery.

In the early era of VATS (video-assisted thoracoscopic surgery), simultaneous bilateral lung resection posed a significant challenge related to prolonged anesthesia, the need for patient repositioning, and the perceived risk of bilateral pulmonary complications. With increasing experience, this approach has been more liberally used safely in low-risk patients undergoing sublobar resection for cancer or bullous disease. ${ }^{2}$ The many advantages include faster overall recovery, lower cost, and greater patient satisfaction. A single subxiphoid incision to simultaneously access both pleural spaces also has been described by several authors for procedures ranging from lobectomy to thymectomy. ${ }^{3,4}$ This approach potentially avoids intercostal nerve impingement, and leads to better cosmesis but requires reorientation with a different view of the anatomy and can be challenging in overweight patients with a high diaphragm.

From a thoracic surgeon's viewpoint, general anesthesia with single lung ventilation is the most convenient approach to perform a lung resection. However, endotracheal intubation is associated with potential for airway trauma, risk to dentition, and side effects of neuromuscular paralysis. Several authors have reported on the successful use of nonintubated thoracic epidural or intravenous analgesia for VATS sublobar resections in healthy patients, as well as those deemed high risk for failure to extubate after surgery. ${ }^{5,6}$ In our own experience, the occasional conduct of nonintubated thoracic surgery requires close cooperation between the surgery and anesthesia teams and willingness to make alterations to well-rehearsed protocols. For this reason, as in the current report, it is beneficial to initially apply this technique in low-risk patients.

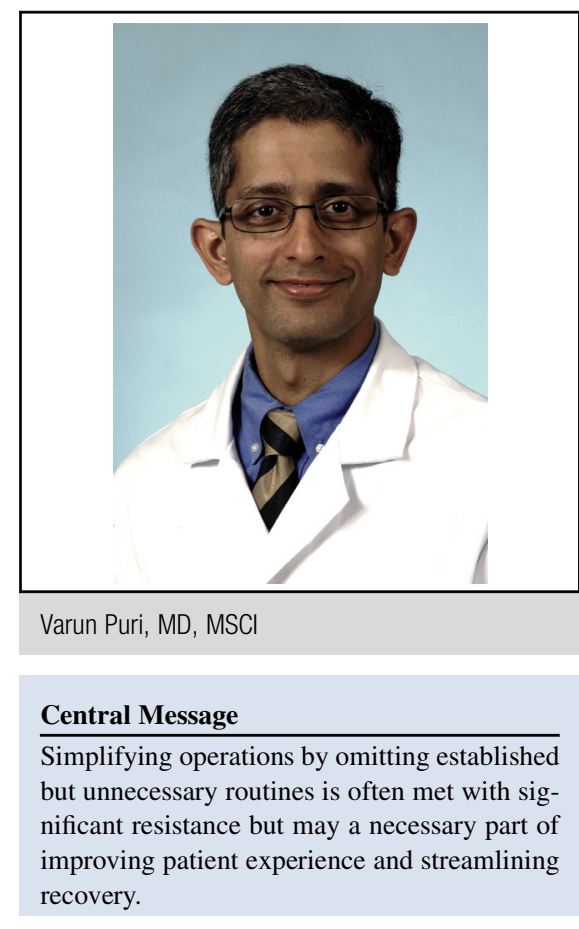

See Article page e115.

The general principles of surgery for bleb-related spontaneous pneumothorax traditionally include blebectomy accompanied by mechanical or chemical pleurodesis (or pleurectomy) and lead to excellent long-term results. However, the pleural intervention (pleurodesis/pleurectomy) is associated with the risk of hemothorax, Horner syndrome, prolonged chest tube drainage, and significant chest wall pain. A recent prospective trial randomized 289 patients with primary spontaneous pneumothorax to either wedge resection and mechanical pleurodesis or wedge resection alone. Intraoperative blood loss and postoperative pleural drainage were lower in the wedge resection-only group, whereas postoperative pneumothorax recurrence rate did not significantly differ between groups. ${ }^{7}$ The authors of the current report have adopted this technique and demonstrated a low rate of recurrence in a recent series. ${ }^{8}$ As cautioned by several authors, it is important to limit bleb resection only (without pleurodesis) to patients with isolated blebs that are completely resected. The recurrence rates are higher in patients with multiple blebs or incompletely resected pathology.

Chest drainage after any thoracoscopic operation, especially lung resection, has been considered a sine qua non in thoracic surgery. Chest tubes, a ubiquitous 
presence on a thoracic surgery service, can be associated with significant neuralgia, tubing and connection-related issues, and iatrogenic pneumothorax at time of removal. A recent report by Lu and colleagues ${ }^{9}$ documented a series of 44 patients in whom chest tubes were omitted after wedge resection. To select a low-risk group, only patients with peripheral lesions, healthy lung parenchyma, no intraoperative air leaks, hemorrhage or effusion accumulation, and no pleural adhesion were included. The pleural space was carefully suctioned out using a nasogastric tube intraoperatively, which was removed when no air leak was seen during lung inflation. None of the patients developed a postoperative pneumothorax. We have similarly selectively omitted chest drainage in our practice in pediatric patients undergoing wedge resection.

Enhanced recovery after surgery (ERAS) refers to the implementation of multimodal pathways aimed at optimizing perioperative outcomes. Intraoperative techniques are eminently modifiable factors that constitute key components of ERAS algorithms. The current report highlights the sequential adoption of surgical techniques that can not only improve patient recovery, but lower costs, and improve patient-reported outcomes. More widespread adoption of such methods, as well as consideration of innovative strategies to challenge the status quo, are the need of the hour.

\section{References}

1. Guo Z, Li S, Yin W, He J. Implementation of a novel enhanced recovery after surgery program in thoracoscopic bilateral bullectomy. J Thorac Cardiovasc Surg. 2017; 153:e115-8.

2. Nakata M, Sawada S, Yamashita M, Saeki H, Kurita A, Takashima S, et al. Surgical treatments for multiple primary adenocarcinoma of the lung. Ann Thorac Surg. 2004;78:1194-9.

3. Han K, Song K, Choi BW. How to develop, validate, and compare clinical prediction models involving radiological parameters: study design and statistical methods. Korean J Radiol. 2016;17:339-50.

4. Zhong Y, Zhou Y, Jiang L, Lin H, Li X, Wen A, et al. Modified transsubxiphoid thoracoscopic extended thymectomy in patients with myasthenia gravis. Thorac Cardiovasc Surg. August 10, 2016 [Epub ahead of print].

5. Iwata Y, Hamai Y, Koyama T. Anesthetic management of nonintubated videoassisted thoracoscopic surgery using epidural anesthesia and dexmedetomidine in three patients with severe respiratory dysfunction. J Anesth. 2016;30:324-7.

6. Hung MH, Liu YJ, Hsu HH, Cheng YJ, Chen JS. Nonintubated video-assisted thoracoscopic surgery for management of indeterminate pulmonary nodules. Ann Transl Med. 2015;3:105.

7. Min X, Huang Y, Yang Y, Chen Y, Cui J, Wang C, et al. Mechanical pleurodesis does not reduce recurrence of spontaneous pneumothorax: a randomized trial. Ann Thorac Surg. 2014;98:1790-6; discussion 1796.

8. Guo Z, Yin W, Wang W, Zhang J, Zhang X, Peng G, et al. Spontaneous ventilation anaesthesia: total intravenous anaesthesia with local anaesthesia or thoracic epidural anaesthesia for thoracoscopic bullectomy. Eur J Cardiothorac Surg. 2016;50:927-32.

9. Lu TY, Chen JX, Chen PR, Lin YS, Chen CK, Kao PY, et al. Evaluation of the necessity for chest drain placement following thoracoscopic wedge resection. Surg Today. September 29, 2016 [Epub ahead of print]. 